\title{
LOS ANGLICISMOS EN ESPAÑNA Y SU PAPEL EN LA LENGUA ORAL
}

La mayor influencia extranjera, durante la Edad Media, en el español, fué la del arabismo'; y la segunda, sobre todo en los siglos xirr, xvirr y xix, la del galicismo2. Ahora predomina la influencia del anglicismo, y aumenta hasta tal punto, que es, posiblemente, el más importante desarrollo lingüístico peninsular del español contemporáneo. A cualquier lector se le ocurrirán anglicismos omitidos en nuestra lista de menos de quinientos ejemplos. Este estudio es fruto de una investigación oral; sin embargo, menciona ejemplos literarios y visuales. Pude explorar poco las áreas técnicas y especiales: el lenguaje de los talleres, de las fábricas y de los laboratorios; de las oficinas importadoras; del transporte marítimo, aéreo y terrestre; de los servicios militares, y de otras especialidades; no obstante, presento ejemplos de casi todas estas categorías.

Esta pequeña investigación, llevada a cabo con una subvención Carnegie mediante la Universidad de William and Mary, habría sido imposible sin la generosa ayuda e interés de muchos españoles. Quiero dar las gracias, entre otros, a los señores don Luis Cortés, don Samuel Gili Gaya y don José Simón Díaz; y, sobre todo, al señor don Emérito Paniagua Comendador, que me regaló cariñosamente su propia lista de anglicismos, y sin cuya ayuda y entusiasmo no habrfa podido escribir este artículo.

1 Con cuatro mil ejemplos, según RAFAEL, LAPESA, Hist. de la leng. esp., 2. ed., Madrid. Escelicer, 1950, p. 97.

3 Véase RAFAel, Baralt, Dicc. de galicismos..., Madrid: Impr. Nacional, 1855; 2." ed., Buenos Aires, 1945. 
Hay pocos estudios sobre el anglicismo en España ${ }^{1}$. En América, donde se emplcan muchísimos más, se han publicado algunos artículos que observan o lamcitan la tendencia; recuérdese la magnnífica obra de Alfaro². En la presente investigación, que trata sólo del español peninsular $y$, sobre todo, del aspecto oral, no he encontrado razones para creer que la abundancia de anglicismos en Hispanoanérica haya facilitado su entrada en España.

He considerado como auglicismos palabras usadas en su forma inglesa o derivadas del inglés; palabras que pasaron de otros idiomas al inglés, y de éste, al español; o bien del inglés al español a través del francés; términos y vocablos creados por gentes de habla inglesa e introducidos en el español: palabras castizas usadas en un sentido inglés (préstamos semánticos o contaminaciones); y traducciones de tropos, complejos $\mathrm{y}$ modismos ingleses.

El anglicismo, que florece ahora en España, no es fenó-

1 Entre las obras que contienen observaciones o listas de anglicisnos: LAPESA, Hist. de la leng. esp., 1950, 2. ${ }^{n}$ ed., pp. 272, 280, 337; Julio Casares, Divertimientos filológicos, Madrid, Espasa-Calpe, 1947. pp. 287-29 I, y Cosas del lenguaje, Madrid, 1943; RAMÓN FRANQUELO X ROMERO, Frases impropias, barbarismos, tolegismos y extranjerismos..., Málaga, Impr. de El Progreso, I9Io; ROBERT K. SPAULDING, How Spanish.Grew. Berkeley. Univ. of Calif. Press, 1948, páginas ro2 y siguientes; y Congreso de estudios sociales. Vocabulario de oficios y profesiones, Escuela social de Madrid, 1946.

2 Ricardo J. Aistaro, Diccionario de anglicismos. Panamá. Im- . prenta Nacional, 1950, 849, páginas. La introducción apareció como. El anglicismo en el español contemporáneo, BICC, IV, 1948, 102-128.

Otros estudios aparecieron en Hispania; véanse los tomos XXIX (1946), 56-66; XXXII (1949), 48-52 y 300-304; XXXIII (r950), 163-I65 y 284-286; XXXIV (1951), 25I-255; XXXVIII (1954), 135140 y 457-459. No he podido examinar los siguientes estudios: LIC. LUIS Cabrera, en Novedades, México, D. P., 22 abril i95I; Peter BoydBowman, A Linguistic Study of the Spanish of Guanajuato, Mexico (tesis de doctorado de Harvard, i949. Debió publicarse en Méjico); JEan Donald Bowen, The Span. of San Antonio, N. M., Univ. of N. M. 1952 y Víctor MANUEx. SUAREz, El esp.que se habla en Yucatan,. I945 (pp. IIO-III). 
meno reciente; hay anglicismos desde la época clásica, como norte, que aparece ya en Covarrubias. Otros ejemplos bastante antiguos son arrurruz (arrowroot), guatarrás (Waiter Ralcigli), pichelingiie (speak English), monis (moneys), ron, bricbarca, sur , este, y oeste. Hay anglicismos, en cambio, novísimos y fruto de modas, ejemplos típicos de manias y aficiones del siglo actual, como charleston, swing, hot, gafas Truman; o bien de uso ocasional o raro, como o.k., all right, hello. En efecto, de cuatrocientos sesenta casos que prescntamos a continuación, tal vez la mayoría sean de empleo aislado o poco frecuente. Algunos, por otra parte, se han incorporado al castellano, como los precitados puntos cardinales, y los siguientes ejemplos: water, lider, reporter(o), smoking, tiuel, balas$t(r) o$, bloc, h. p., box-calf, guasin, crismas, trolebis, bar, bebé, revólver, rifle, turista.

E1 anglicismo ha penctrado en España por medio de la literatura, la guerra, el mar, los viajeros, el comercio, los deportes, el cine, el periodismo, la tecnología y la ciencia; en la época reciente, sobre todo, por los deportes, el periodismo, las traducciones populares, el cine, el comercio, la tecuología y la ciencia. El periodismo desempeña un importante papel; además del número notable de anglicismos que aparecen en los periódicos, se difunden en un área muy amplia. El periodismo absorbe fácilmente muchos anglicismos, con las traducciones rápidas y descuidadas de los despachos repartidos por los tres grandes servicios anglosajones de noticias ${ }^{1}$. Fintre los innumerables anglicismos que se pueden citar de periódicos, he aqui, a título de muestra, algunos ejemplos: la bandera americana (por norteamericana) América (por Norteamérica, Estados Unidos), la guerra fría, la cortina de hierro, en tiempo récord, bird-watchers, night club, bar, barman, penally y posiblemente próximo oriente.

En tres o cuatro traducciones populares tomadas a título de ejemplo, he hallado los siguientes anglicismos: chances,

1 Como dice AIfARO, Dicc. de angl., p. Io. 
aprobar de (por aprobar a), culi, tiempo de depresión (por crisis económica), madera de teak (por teca), Christian Science, brogue. Tal vez la mitad de los títulos que se ven en algunas librerías sean de obras traducidas del inglés. Aunque menos importante que el periodismo, este vehículo debe de influir también en un público bastante extenso.

El impacto del cine norteamericano en España es grande. Probablemcnte entrarían más anglicismos en la época de las películas habladas cn inglés; pero aun con el español castizo de las películas dobladas, se conservan en inglés algunas palabras, canciones, rótulos y letreros. $Y$ las películas tienden a despertar el interés por la cultura anglosajona y facilitan así la adopción de anglicismos. Cowboy, gunman, gang y ganster, o. k., pueden deberse en parte, al menos, a la:s películas. Se habla de una Hilda, de un Tyron Power, como tipos. Del vocabulario cinematográfico se han adoptado hablar en off, make-up, set (decoración), astro y estrella, trailer, film y filmar.

E1 turismo de españoles, que favorecería los anglicismos entre la gente de buen tono de hace treinta o cuarenta años, según Marquina y otros autores de la época, tiene hoy poca importancia. El turismo de norteamericanos en España, antes insignificante, aumenta cada vez más y facilitará la introducción de muchos anglicismos.

El comercio de postguerra ha contribuído a ese proceso. En los anuncios abundan formas inglesas. Donde antes se decía "descapotable", ahora anuncian también convertibles. Fajas Sportex, insecticida Cam-spray, acondicionamiento de aire, prendas de nylon, films de gansters, frigidaires, pick-ups, un quemador-grill radiante, persianas venecianas, y transistors se encuentran en los reclamos. En la peletería, se habla de skunk (mofeta) y de opossum (zarigüicya). En los escaparates se pueden ver y comprar trench coats y trincheras, un juego llamado Play-ball, "frontón sin pared", gabardinas, un estic para el hockey, clips, blocs o bloques y otros artículos exhibidos con nombres tomados del inglés. En las grandes casas importadoras y cn sucursales norteamericanas e inglesas (Interna- 
tion Cash Register y Westinghouse, etc.) el personal adopta y se familiariza con expresiones y términos ingleses, seguin me han informado. Varios productos ingleses y norteamericanos han penetrado en el habla común. Entre las marcas cxtranjeras de forma inglesa, nos interesan sólo las que se generalizan, como palbich (un tejido, del ing1. Palm Beach), delco (cualquier distribuidor), gilette (cualquier hoja de afeitar), cárter (encaje de motor), flit (insecticida rociada), claxon (bocina de coche), kódak (pequeña cámara fotográfica), frigidaire (refrigeradora), colt (pistola automática), bessemer (convertidor), pullman (vagón de lujo). Las invenciones han creado portland y portlandista, teléfono, rifle, revólver, transistor, pick$u p$, y fonógrafo. De razas de animales domésticos tenemos la gallina leghorn (de Liorna, en ital., Livorno), y varios perros; dogo, bulldog, sétter, cócker, póinter, fox-terrier; de plantas, la naranja washington o guasin; y se anunció una flor, zinnia fantasy. Bastantes empresas llevan nombres ingleses o tomados de los países de habla inglesa, como Bar Arizona, Bar Sport Miami Restaurant, y café Miami, el Manhattan, el California, dulcería Texas y Bar Texas, peluquería de señoras Betty, $P a$ lace Hotel, Family Hotel, Derby Sastrería, Caza-Sport, un cine el Capitol, y una zapatería Boston. Estos nombres se ellcuentran en las grandes ciudades o en las de veraneo, como San Sebastián.

Los deportes han favorecido mucho los anglicismos. Del golf proceden drive, links, iron, caddie, hazard, tee, brassie, niblick, putter, green, clubs, y otros. Los aficionados son, en principio, gente rica que ya tiene bastante familiaridad con $\mathrm{cl}$ inglés, lengua que desconoce el español medio. El vocabulario del fritbol, en cambio, está mejor incorporado al habla común, con chut y chutar, offside, gol y golaverage, hutra, córner, back, etcétera. Otros deportes salpicados de anglicismos: el boxeo (ring, knockout o k. o., wélter, fault, groggy, upper-cut, etc.); la natación (crawl); carreras y saltos o el track (cross-country o cros, sprint, cubrir una distancia, batir el récord); carreras de caballos (handicap, jockey, steeplechase, turf, match, pony, rally-paper); el hockey (stick o estic); el tenis (smash, game); 
el ping-pong; el poker (bluff, deuce); la pesca (carpa royal), el bridge (slam, rubber); el automovilismo (raylle), presumiblemente los yates, y otros deportes y juegos. Es natural que los deportes y recreos tradicionales, como los gallos, los toros, el jai alai, carezcan de anglicismos.

El mar, medio tradicional de actividad inglesa y española, ha proporcionado al español términos náuticos ingleses. He aquí una lista de épocas y frecuencia muy variadas: spinnaker, boya, cliper, eslinga, bricbarca, bote, pailebote (de pilot's boat), yola (yawl) yate (yacht), bauprés, madera de teak (teca), outrigger y del vocabulario naval, ejemplos seguros o probables son: guardacostas (coast guard), destructor (destroyer), buque de desembarco (landing craft), monitor, portaaviones. Habrá muchos anglicismos en la terminología ingeniera marítima $\mathrm{y}$ naval, campos que desconozco desgraciadamente.

La técnica y la ciencia modernas son las áreas de mayor número de anglicismos en el idioma. Sería imposible presentar una lista completa de tales términos, que, por otra parte, no han pasado, salvo algunos casos, al habla común. Los ejemplos que cito como muestras ilustran esta importante esfera de anglicismos.

En la medicina, se emplean antilistamina, auromicina, crush sindrome, estreptomicina, penicilina, mal de Pott, electroshock, y shock traumático. Otros términos técnicos o cientificos son transistor, ciclotrón, ciclon, relay, shunt, y las abreviaturas SOL, CONSOL, LORAN, RADAR, y SONAR. Se han propuesto también, como anglicismos probables, aerodinámico, amplificación, audiofrecuencia, interferencia, micrófono, microsurco, resistencia, televisión, transformador, transmisor, esterioscópico y cronómetro, aunque no están confirmados.

Los nombres propios tienen poca importancia en los anglicismos. Ya he citado algunos términos derivados de nombres propios: cárter, bessemer, pullman, mal de Pott, morse, boicot; hay empresas que llevan nombres propios (peluquería Betty, Bar Texas, etc.); personalidades (un Tyron Power, gafas Truman). En los diccionarios figuran las personificaciones John Bull y Shylock. Algún nombre de perro o de persona (Bobby). 
es inglés. A diferencia de los pueblos anglosajoiles, lo: españoles no emplean nombres extranjeros sino tradicionale:s para las niñas.

Algunos anglicismos han llegado a través de otro; idionias. Ciertas palabras exóticas penetraron, princro, en el inglés, y establecidas en él, pasaron, luego, al castellano: culí, champí, jungla (del hindustani); gong (del malayo); términus, alma mater, stadium, aquarium (del latín); dril (del alemán drillich, del latín trilix), bauprés (ingl. bowsprit, antiguo ingl. jouspret del holandés boesgspriet; a través del fr. beaupré), y premic\% ("el premier británico»), plató, turista, y convoy (del francés). Control se estableció en España en el sentido francés de "onnprobación, inspección», y volvió modernamente a rntiar con el del inglés "dominio, dirección".

Varios anglicismos revelan, por su aspecto, una citiclutanía secundaria francesa: bloc, paquebote, bebé, comité, rallye, bitlce. redingote, confort, esplin, bauprés, sidecar, autocar. Fstos pre:tamos son de los que penetraron en España por vía francesi.

La existencia de extranjerismos no implica, nscesariamente, imitación sin motivo. Los jréstamos pucden llenar un hueco; por ej.: expresar un concepto nuevo; despertar la fantasía popular por lo pintoresco; o imponerse pror el prestigio.

Sobre todo, las ciencias y la tecnologia, y algunos deportes introdujeron en España objetos, ideas y conceptos nuevos que necesitaban términos también nuevos, para lo cual era más fácil adoptar, hispanizar, o traducir una expresión extranjera que buscar un vocablo castizo. Además de los términos técnicos, algunos anglicismos útiles pueden ser boy-scout. detective, eslinga, esterlina, rotario, standard, jeep, jockey, hobby, morse. Palabras de aspecto pintoresco: sheriff, cowboy, living hall, clown, boss, ganster, flirt. Como palabras prestiriusas citemos slip, office, magazine, colcrén, weekend, lunch, pony, sandisich, nurse.

Se nota también algún eufemismo, útil para evitar palabras groseras, como wáter, bar (más elçrante que cantina $y$ taberna). Hay varios otros. El motiro puede ser complejo; 
jecp presenta el aspecto util (coche de tipo nuevo), el prestigioso (producto de la inventiva norteamericana, y por las bucnas calidades da la máquina misma), al mismo tiempo que es palabra pintoresca.

Por último la imitación está favorecida a veces por la mera presencia de la forma inglesa en los partes y libros que se traducen, en las películas y los noticiarios que se doblan; por razones de humorismo, de elegancia, de pura utilidad, se insinúa la palabra en la conversación.

Algunos préstamos fueron dislocados y reemplazados por formas castizas. A speaker y a broadcasting de los primeros años de la radio se prefirieron luego locutor y radiodifusión. Con la popularización del atletismo en España, se adoptó sport; pero, luego, se recordó el antiguo deportar y de alí deporte, que ha tendido, después, a reemplazar a sport.

El anglicismo puede constar de una o más palabras ingle sas hispanizadas o no; o tomar la forma de palabras españo las adaptadas a un sentido inglés o que traducen un tropo'modismo, o complejo inglés.

La palabra inglesa cambia poco o mucho su pronunciació $n$ según entre por vía oral o visual y la dificultad, para el español, de sus fonemas. En principio, el español sustituye el fonema inglés por el que le corresponde o le parece más pronunciable. Las consonantes inglesas $b$ y $v, c^{e}$ y $c^{i}, d, g, j, l, n, p$, $r, s, t, y$ y $z$, tal vez las más diferentes en inglés, asumen la forma española; bateador, vamp, magazín, office (ofiz), girl, jersey, lady, ténder, pickpocket, repórter, este (= east), recital, box-calf (pronúnciase boscal), yate, búlldozer.

No se pronuncia, a veces, la $h$, al menos entre el pueblo, hobby, hall, hockey. I, a w puede pronunciarse de varios modos, según la época y el nivel de la adopción; gu-, como en la antigua lengua romance de occidente (cotéjense guardia, guisa, guerra): guatarrás (Walter Raleigh), guasin (Washington); como $w$-, $0-$, o $v$ - (por influencia probable del intermedio frailcés) en wáter, oeste (west), vagón (wagon). La -w-interna se asemeja a la $u$ o a la $v$ seguidas de vocal: suéter (sweater) 
Browning (pron. brovin), broadway (pron. bróvai). Caso curioso es el de cowboy (pron. comboy).

No se efectuan todas las semejanzas nás próximas, sin duda por influencia de la ortografía. Por ejemplo, la $j$ sucle pronunciarse a la castellana (jersey, jockey, jungla), aunque la $y$ suena más cerca de la $j$ inglesa. La $c^{b}, c^{i}$, no conserva su valor de $s$, sino que se castellaniza: office (ofiz), penicilina. Ia $s h$, sin equivalente, toma la forma de la ch en los préstamos populares: champu, chutar.

Como la vocal española difiere, en principio, de la inglesa, todas éstas, en los préstamos, toman el matiz español: bate (bat), este (east), míster y sidecar (en ingl., sáid-), office, ron (rum), etc.

En algunos préstamos, el inglés sirve principalmente de base para un sufijo español: boxeador, boicotear, golear, goleada (del inglés goal).

Se añade a veces una vocal, antes o después de consonartes dificiles: estic, esterlina, esnob; norte, oeste, este, yate, bote; aunque no siempre (sport). Se conserva modernamente el plural inglés en -s en lugar del español -es después de consonante aunque resulta difícil de pronunciar: transistors, handicaps, slacks. A veces esto es posible por omitirse la consonante final: clips (pronúnciase clis), pick-ups (picris), round (raun), boxcalf (boscal). No se pronuncian tampoco consonantes difíciles en el interior de la palabra: leggings (leguis), palbich (de Palm Beach), gunman (giman), Browning (brovin).

Casos de desplazamiento acentual sobre todo en los préstamos visuales: tractor (de tráctor), sidecar (de sidecar), recital (recital).

E1 anglicismo puede ser oral o visual: aunque no se distinguen completamente estas dos categorías; el aspecto visual influye sobre el préstamo oral, y el concepto de la pronunciación inglesa, en la forma visual. Ejenuplos visuales parecen ser morse, nurse, puzzle, recital, jersey, fade-out (fadeút); formas orales: guatarrás y pichelingiie (arcaicas), pailebote, suéter, ponche (ingl. punch.).

A veces reconstruímos una forma española a partir de una 
inglesa, aunque esto cs raro, posiblemente aparcar y aparcamiento, a basse de parque + prak y parking; cxplotar, por influencia del inglés, explode, junto con la analogía de cxplosión; fertilizar, de fértil con la analogía del ingl. fertilize.

Un préstamo de forma conveniente adquiere la hispánica sólo con alguna modificación en la ortografía y un sufijo apropiado: reportero (de reporter), pionero (de pioneer), eslinga (de sling), ponche (de punch), transformador (de transformer), teléfono (de telephonc), factoría (de factory).

De los anglicismos formados por palabras castizas, o sea los préstamos semánticos, el más sencillo es la contaminación, en que el vocablo castizo amplía su sentido con el inglés. Así, educación (abarca ahora el sentido inglés de instrucción), tráfico (con el sentido inglés de circulación), carro (de la máquina de escribir, del inglés carriage).

Merecen destacarse las traducciones de tropos, modismos, y complejos ingleses, que suelen tomar estas formas:

a) 'Tropos: astro y estrella; purga (política); as (campeón).

b) Neologismos modelados sobre neologismos ingleses: $r e-$ frigcradora (de acterdo con refrigerator), rascacielos (traduce slyscraper), limpiaparabrisas (voindshield-or vinsdcreen-viper), lanzacohetes (rocket-launcher), pluma fuente (fountainpen, forma rara en España).

c) Traducciones de modismos y complejos ingleses: hacha... enterrada (bury the hatchet), teléfono de larga distancia (longdistance telephone), casa remolque (house trailer), dibujos animados (animated cartoons), acondicionamiento de aire (air-conditioning), estación de servicio (service station). Es difícil no ver en estas expresiones el probable modelo inglés.

Si el préstamo no es término técnico ni científico, fácilmente su sentido cambia o se restringe. Ejemplos de este último caso: competición (en los deportes; en ingl., competencia, concurrencia en general), balast( $r$ ) o (de la vía férrea; en inglés, peso para estabilizar, por ej., lastre); dumping (inundación económica; en ingl., descargo en general,) dogo (raza de perros: en ingl., perro), groggy (en el boxeo; en ingl. borracho, que se tambalea), lider (político; en ingl., jefe, caudillo, el que 
manda o conduce), eslinga (aparato de buque; en ingl., también, honda, cabestrillo, braga, etc.), recital (de música; en inglés, también narración), bloc (taco de hojas de papel; en inglés, manzana de casas, conjunto, bloque; etc.); fading (de radio; en ingl., desvanecimiento). Otra forma de limitación es la elipsis: crismas (tarjeta de pascua; del ingl., Christmas card), office (diplomático británico; en ingl., despacho), trinchera (de trench, trinchera, + coat, impermeable).

Ejemplos de cambio de sentido: pullman (vagón de lujo; en ingl., coche -cama), esnob (amigo de novedades: en ingl., fachendoso, presumido), gabardina (tipo de impermeable; en inglés, la tela), camping (merienda al aire libre; en ingl., vida al aire libre o en tienda), ticket (rccibo, etc., en ingl., principalmente billete de entrada o de viaje).

Varios préstamos han entrado como iniciales O.K., FBI, y los términos técnicos LORAN, RADAR, SOL, CONSOL; o bien han tomado forma de iniciales; tobralco (de Total Broadhurst tee Co.), k.o. (de knock-out). ${ }^{1}$

Pocas huellas se encuentran, en los anglicismos, de la sintaxis inglesa. Algún orden de palabras o empleo adjetival de substantivos en frases hechas (Real Madrid Club de Fitbol, Real Automóvil Club); tal vez en un ejemplo ya citado, aprobar de, y en el orden de un anuncio que reza: «señora, haga su hogar confortablen. En principio, la sintaxis no tiene importancia para los anglicismos.

I.os préstamos han podido penetrar en España de Inglaterra o de Norteamérica. De los ingleses vinicron probablemente: balast( $r$ )o, portland, sétter y las razas de pcrios, cheque, gentleman, nurse, folklore, camping, sprint, hanaicap, jockey $\mathrm{y}$ la mayoría de los términos deportivos, jersey, boycot, esnob, crush sindrome, mal de Pott, tranvia, esterlina.

$\mathrm{Y}$ de los norteamericanos con probabilidad: coivboy, boss,

1 Esta sección sobre las formas de los anglicismos debe mucho a la inspiración del articulo de Spencer L. MURPHX, Jr., Notes on Anglicismos in American Spanish, Hispania, XXXVIII (1954), 457-459. 
gang, y ganster, gunman, delco, morse, hot, swing, baloncesto, baseball, sheriff, broadivay, revólver, rifle, teléfono, slacks, nylon, slip, palbich, suétcr, film, cliper, rotario, y muchísimos términos técnicos. En bastantes casos sería difícil distinguir el origen británico o norteamericano de un préstamo. De estas influencias, predomina alora la norteamericana; antes, la inglesa.

Recordemos que el comercio entre las leinguas de Shakespeare y de Cervantes es recíproco; se ha estudiado la influencia del español en el inglés, comparable con la del inglés en el español ${ }^{1}$. Sería interesante contrastar la naturaleza de las dos influencias, que, por otra parte, alcanzan mayor desarro110 en América, entre las culturas colinjantes, que en Europa. En España, sin er `argo, aumentará seguramente este desarrollo por la simpatía entre los pueblos español y norteamericano, y el auge de su comercio mutuo y de sus contactos culturales y militares.

\section{ANGLICISMOS PENINSULARES:}

Símbolos empleados:

? anglicismo probable.

() anglicismo sin comprobar en España.

p. documentado en un periódico.

tr. documentado en una traducción de novela.

$\mathrm{V}$ mencionado como anglicismo en $V o x$, dicc., gen. ilustr. de la leng. esp., 2. ed. por Samuer Gili Gaya, Barcelona Spes., 1953 .

D mencionado en forma francesa como anglicismo por ALBERT DAUzAT: Dictionnaire étymologique, París, Larousse, 1938 .

1 HaRold W. BExtrer, Dictionary of Spanish Terms in English. New York, 1932. 
i comunicado por un informador español.

fr. entró por vía del francés.

c. oído en la conversación, en España.

acondicionamiento de aire (airconditioning), p.

aerodinámico, -a (subs., adj.) ?. all right (poco usado), i.

alua mater (es decir, la universidad de uno), $p$.

América (por Estados Unidos), $\mathrm{p}$.

American dancing, $i$.

americano (por norteamericano), p., c.

amplificación, ?.

animador de dibujos, ?.

antihistamina, c.

aparcamiento, -mento, $\mathrm{p}$.

aparcar, c.

aprobar de (por aprobar a), tr.

arrurruz (arrowroot), V.

as (ace, sobresaliente, campeón), p.

astro (de cine, teatro, etc.), i.

Athletic (nombre de un club), i. andiofrecuencia, ?.

auromicina, i.

autocar fr., V.

auto-radios, ?, p.

back de fútbol, i.

balast(ro), V, i.

balastrera, i.

baloncesto (basketball), V.

balonmano (handball), i.

baseball, V.

basketball, i.

bate, bateador, i. batir: véase récord.

bauprés fr. (bousprit, ingl. medio, bouspret; holandés, boegspriet), D.

bebé fr., $D$.

bessemer (convertidor), V.

Betty (empresa).

bill de indemnidad ${ }^{1}$.

bird-watchers, $p$.

bitters, i.

bloc, fr., D.

bluff, $\mathrm{V}$.

Bobby (nombre de perro, c; nombre de persona, p).

bodas de plata, de oro, de diamante, ?, p. (silver, gold, dictmond redding anniversary).

boicot, boicoteo, boicotear, $V$.

boogie-woogie (nuls. de baile), i. boom (prosperidad, ange), i.

boss, i.

Boston (empresa).

bote (boat), V.

Bowling (empresa).

box-calf, c.

boxeo, boxear, boxealor, V.

boy (joven cle teatro), i.

boya, $\mathrm{V}$.

boy-scout, i.

brassie (del goll), i.

break, V, i.

breeches ${ }^{2}$.

bricbarca, V.

bridge, $\mathrm{V}, \mathrm{i}$.

1 Bill de indemnidad: véase MARIANo DE CAVIA, Limpio y fijo, página 29.

2 Breeches: véase MaRIano de Cavia, Limpio y fijo, p. 25. 
broadcasting ${ }^{1}$, $\mathrm{i}$.

broadway, i.

brogue (acento irlandés), tr.

budin (pudding), $V$.

Bull: véase John Buil.

bulldog, Pequeño Larousse.

bulldozer, i.

bully (término del hockey), i.

buque (o, barco) de desembarco, $\mathrm{V}, \mathrm{p}$.

caddie (del'golf), $i$.

cafetería, p.

cake-walk (un baile), i.

cámara fotográfica (del ital. e ingl.), ?.

cameraman, $i$.

camping (merienda de campo), i.

cam-spray (un producto anunciado), p.

cap (una bebida), i.

Capitol (empresa).

carro (de máq. de escr.), ?, i, p.

carro (por coche automóvil), i.

cárter, c.

casa remolque (trad. de housc-

trailer), ?.

catch-as-catch-can (boxeo), i.

Caza-Sport (empresa).

ciclón, y anticiclón, fr., D.

ciclotrón.

citty, i.

cleek (del golf), i.

clip, c.

cliper, V, p.

close-up, i.

clown, i.

club, V.

clubs (del golf), i. cócker, c.

cóctel, i, V.

cok-carbones, pi carbón de co

(ingl., coke), i.

colcrén (coldcream), c, p.

colt (pistola autom.), $\mathrm{i}$.

comité, fr., V.

competición, ?.

confort, fr.

confortable, fr.

CONSOL (término, abrev.), i.

contacto (aviación), i.

contract bridge, $i$.

control, controlar (dominar), p.

convertible (por descapotable), p.

convoy, ?.

córner (en el fútbol), $\mathrm{V}$, i.

cortina de hierro (trad. de iron curtain), $\mathrm{p}$.

cosechadora (trad. de harvestter), c.

cowboy, i.

crawl (natación), i.

cricket, i.

crismas (tarjeta de pascuas), i.

cronónetro, ?.

croquet, V, i.

cros, cross, cross-country, i, p.

crush sindrome (s. de aplastamiento), $i$.

cubrir (por recorrer una distancia), $\mathrm{p}$.

cuchillos largos (los blancos, en una película de indios).

culi, tr.

cliampú (de shampoo, del hindustani).

chance (suerte, posibilidades), tr.

1 Broadcasting: véase Julio Casarss, Cosas del lenguaje, III, reemplazado por radiodifusión, según me comunicó el señor GirI GaYA. 
charleston (un baile), i.

Christian Scicnce, tr.

chut, chutar (ile shoot en el fútbol), i.

dancing (baile), i.

dandy, dandismo ${ }^{1}$.

delco (por distribuidor), c.

depresión (por crisis econl.), tr.

Derby Sastreria (empresa).

destructor.

detectar ${ }^{2}$.

detective, $\mathrm{V}$.

detector, ?.

deuce (deJ póker), i

dibujos animados (animated cartoons), ?.

dogo, V.

dribbling (del baloncesto), i.

dril, V.

clrive (del golf), $i$.

driver (del golf), $i$.

dumping (inundación econ.), i.

educación (por instrucción), i, p.

electric-shock, i.

entrenar ${ }^{3}$

eslinga, $V$.

eslip, slip (combinación), i.

esnob, esnobismo (en ingl., fachendoso, presumido), V.

esplín (de spleen, mal huunor, ma-

licia), V.

estación de ferrocarril, ?.

estación de servicio (por ej., en

Madrid, cerca de Fern. VI). este, V.

esterioscópico, ?.

esterlina, $c, \mathrm{~V}$.

estic, stic (del hockey), c. estrclla (de cine, etc.), i.

estreptomicina, $i$.

explotar (por hacer explosión, estallar), $p$.

express, expreso: tren, c; café express.

factoria, $c, p, V$.

fade-out, i.

facling (de radio), i.

Iamily Hotel (cmpresa).

fantasy: "zinnia fantasy» (nuera variedad anunciada).

fault (del boreo), i.

IPBI (Oficina federal de investigaciones), i.

fertilizar (por abonar), ?, i.

film, filmar, p, c.

fin de scmana (trad. de werkend).

firma, fr.

five o'clock tea, $p$.

flash (en la radiodifusión), i.

flirteo, ilirtear, V.

flit (insecticida rociada), i.

flow (¿de la telcvisión, ?), i.

folklore, folklórico, folklorista, folklorisno, $V$.

fonógrafo.

fotocolorímetro, ?. c.

fox swing (un bailc), i.

fox-terrier, c.

free hit (golpe franco, deportes), i.

free kick (del fútbol), i.

frigidaire, $p, i$.

fútbol, $i, p, V$.

futbolin (juego inccálico).

gabardina (de gabardine, cierta tela), fr..

1 Dandy, dandismo, véase Spaulding, How Span. Grew.

2 Anglicismo probable, según me comunicó el señor Gili Gaya.

3 Entrenar, véase SARAr.EGUI y Medrna, Escarceos filológicos, página 273 . 
game (de tenis), $i$.

gang, gangster o ganster, $p, i$.

garden-party (merienda al aire

libre; en ing., fiesta de jar-

dín), i.

gasoil, c.

sprint: gaveta sprint (vehiculo). gentleman, i.

gilctte (hoja de afeitar), $i$.

gimnasticlub, $i$.

gin-fizz (cierta bebida), i.

girl (chica modernisima; en ing., uuchacha, niña), c.

gol (de fútbol, etc.; ingl., goal), $\mathrm{V}$.

golaverage (promedio de goles), ?, i.

golear, goleada, V.

golf, i, p.

gol-keeper (guardameta), i.

gol kick, i.

gong (del malayo, por via del inglés), p.

gramófono.

grill (quemador-grill radiante), $p$. groggy (en el boxeo), i.

green (del golf), $i$.

guardacostas (trad. de coast guard), p.

guasin: véase wasinton.

guatarrás (inglés, de Walter $R a$ leigh), i.

guerra frla (trad. de cold war), p.

gúnman, $i$.

hacha... enterrada (trad. de «bury the hatchet"), tr.

hall, c, V.

handball, i.

handicap (en las carreras), $p, i$.

hazards (del golf, $i$.

hello (poco usado), i.

high-life, $i$.

hobby, i.

hockey, i, p. hot (cálido), i.

Floricla: Hotel (empresa).

h. p. (horscpower: caballos), c, p. hurra (grito en el fútbol), $i$.

inferiority complex (complejo de inferioridad), comunicado por el señor Gili Gaya.

interferencia (esp. radio), ?, i.

interviú, V.

iron (del golf; club), i.

jeep, p.

jersey, c.

jockey, $V$.

John Bull (personif. de Inglaterra), V.

jungla (del hinclustani), c.

Júpiter: por - (trad. de By Jove): tr.

kickoff (del fútbol), i.

kit (del ing. radio $k i t$ ), i.

knock-out, k. o., i, p.

kodlak (por cámara fotog.), $i$.

lady, $i$.

larga distancia (teléfono de, trad. de long-distance telephone), ?.

lawn-tennis, $i, \mathrm{~V}$.

leggings, $i$.

leghorn, c, p.

lider (leadcr), p, V.

links (campo de golf), i.

linólco, $\mathrm{p}$.

(living) (sala, esp. en Hisp-Am.).

(lobbying, lobbyist).

lock-out, i, V.

long-playing (discos - ), c.

looping, $c$.

I,ORAN (abr. de long range navigation).

lord, pl. lores, $\mathrm{p}, \mathrm{V}$.

I.td. (abr. de limited; sociedad limitada: Sanz y Ferrer, Ltd., de Bilbao).

luna de micl (trad. de honeymoon) lit. 
luuch (agasajo; en ingl., almuerzo), i, p, V.

macadán, macadaun; macadamizar (macadam, del inventor McAdam) i, V.

magazine (revista), i.

make-up (maquillaje; cinem.), i. mal de Pott (med.), i.

mambrú (de Malborough; canción), i (y chimenea de buque), $\mathrm{V}$.

manager (gerente), i.

manejar (por manejarse), ?, tr. Manhattan (empresa), i.

match (deportes, esp. boxeo), i, V. meple (arce), ?, lit.

Miami Restaurant (empresa).

micrófono, ?, i.

miss (de concurso de belleza; en inglés, señorita), $i$.

mister (abr. Mr., título; y subs., aunque esto es incorrecto en inglés), $i$.

(uroni) (de money), V.

monis (de money, dinero), V.

inonitor (buque de guerra).

inorse (del inventor Morse), $\mathrm{V}, \mathrm{i}$.

Mrs. (abr. de Mistress, pron. missis: señora), i.

niblick (del goif: club), i.

nickey (¿especie de jersey?), ?, i. norte (north), V.

Nueva York: Hotel - (empresa). nurse (niñera), i.

nylon (pron. náilon como en inglés, y nilón), c, i, p.

oeste (de west), V.

off: hablar en - (término cinematográfico), $i$.

office (ministerio británico de Asuntos Extranjeros; de Foreign Office), i. off-side (deportes: fuera de los limites), i.

o. k. (está bien, bueno), i, c.

opossum (zarigüeya; en la peletería, piel de), c.

órsai: véase off-side.

padre: dia del —, ?, p.

pailebot, -bote (de pilot's boat) $Y$.

Palace: Hotel - (empresa).

paquebote (de paquet-boat), fr.

penalty (deportes), $\mathrm{p}$.

penicilina, $i$.

penique (penny), p, V.

performance, $i$.

pichelingüe (pirata; antic.; de speak English; comunicado por el señor Luis Cortés).

pickles Peq. Lar.

pickpocket Peq. Lar.

pick-up, i, p.

picnic (meriencla al aire libre), i.

(ping-pong), (juego), i.

pionero (pioneer), i.

pipeline ${ }^{1}$.

plató (término cinem., de plateau, del fr. plateau), fr., i.

playback (aparato para tocar lo grabado), i.

play-ball (emprender la jugada o como aviso: ¡listo! marca de cierto juego).

(pluma fuente), i.

póinter (raza de perros), i.

póker, i.

ponche (de punch, puñetazo y bebida), i, V.

pony, i.

(pool), i.

portaaviones (¿trad. de airplanecarricr?), ifr.?

portland $i, V$; portlandista, $i$.

1 Pipeline: véase J. CASAREs, Cosas del lenguaje, III. 
porto-flip (bebida; de flip, nounbre de cierta bebida), i. premier (cl - británico), F. próximo oriente (¿trad. de Near East?), ?, p.

pudding, pudin, $i$.

pudinga (de pudding), minerol.: cierta roca, $V$.

pull-push (término de radio), i. puntos (por tantos: tenis), tr. purga (polltica), ?, i. putter (del golf), i. puzzle (acertijo, etc.), i. quaker (cuáquer oats, avena machada), i.

Racing (cierto club de fútbol), i. RADAR (abr. de Radio detection and range), $\mathrm{i}, \mathrm{V}$.

Radio Gaceta (cierto periódico), ?. raid (vuelo de récord; de raid, incursión, o bien de ride, cabalgada), $i$.

rail (carril, riel), $i$.

rally-paper (en las carreras), i. rallye (prueba automovilistica; de rally-paper wediante el fr.), fr., $p$.

rascacielos (trad. de skyscraper). Real Automóvil Club, ?, p.

recital, i.

reconversión (sugerido por el señor Gili Gaya).

récord, batir el récord, $i, p$.

recordman (el que tiene el récord

o la marca), i.

redingote (de riding coat, me-

diante el fr.), Dicc. de la Acad. referec (deportes: árbitro), i.

refrigeradora (trad. de refrigerator), p, i.

rclay (clectr.), i.

reportaje (de reporter), ?, p.

repórter $\mathrm{p}, \mathrm{y}$ reportero.

resistencia (electr.), ?, i.

revólver, $\mathrm{V}$.

rifle, c, V.

ring (del boxeo), p, i.

ron (de rum), $\mathrm{V}$.

rosbif 1 .

rotario, rotarismo.

round (del boxeo), i.

royal: carpa royal, $\mathrm{p}$.

rubber (del bridge), i.

rugby, $i, V$.

sandwich, V, c.

scooter, p.

score, tr.

script (término cinem.), ?, i.

season ${ }^{2}$.

semifinal (deportes), p.

set (decoración, término cinema-

tográfico), $\mathrm{i}$.

set (partida de tenis), $i$.

sétter (raza de perros), i.

sex-appeal (atracción sexual), i.

sheriff, i.

shock ${ }^{3}$.

shock traumático (med.), i.

shocking (escandaloso), i.

shoot: véase chut.

slow (espectáculo), i.

shunt (puente eléctrico), i.

Shylock (personificación del usurero), V.

I Véase Spauiding, How Span. Grew.

2 Season: véase SARAlegui y MIEdina, Escarceos filológicos, pá$\operatorname{sina} 273$.

3 Shock: véase J. CaSARES, Cosas del lenguaje, p. 98. 
sidecar, c.

"Singing in the rain" (título de canción: Cantando en la lluvia), ?, i.

skunk (mofeta, en la peletería), c. slacks (pantalones sueltos), i.

slam, eslam (de tenis o bridge), tr.

sleeping, ?, i.

sleeping-tabla (vulgarismo jocoso por vagón de tercera), i.

slip: véase eslip.

slogan, eslogan (lema, mote en la propaganda), p.

smash (saque en el tenis), i.

smoking, fr., $V$.

SOL (abr. de un sistema de aterrizaje), $i$.

SONAR (abr. de un sistema de navegación y detector), i.

speaker (locutor, que lo reemplaza), $i$.

speech (¿discurso?), ?, i.

spinnakker (cierta vela de yate), tr.

sport, muchos ejemplos, y V; caza-sport (empresa).

Sportex: fajas - (marca de una prenda), $p$.

sportsman, p; sportman (error de ortogr.), $\mathrm{V}$.

spray: cam-spray (marca de insecticida), $p$.

sprint, sprinting; carrera al esprint, i; gaveta sprint, marca de triciclo.

stadium, ?, i.

stand, $p, i$.

standard (en cierta obra médica; - de vida, nivel de la vida; servicio - letrero; Standard Eléctrica, S. A.; y otros ejemplos), i.

standardizer, i. star (de cine, etc.), ?, i.

"Star" Armas (empresa).

steeplechase (deportc), ?, i.

stick: véase estic.

stock (surtido), i.

stop (alto, deténgase), ?, i.

street (calle), ?, i.

suéter (sweater), i, c.

sur (south, ant. ingl. suth), V.

swing (cierta música; adj.: ch́lido), i.

teak (por teca), tr.

tee (del golf), i.

teléfono.

televisión, $\mathrm{c}$.

telón de bambú (de acero - trad. de bamboo curtain, iron curtain, sentido pol.), i.

ténder, i, V.

tenis, V.

términus: Hotel - (cmpresa), D.

ternos, sing. pop. termo: Vox (thermos bottle), ?.

Texas: confitería y bar - (empresas).

ticket (recibo, talon, etc.), c, V. tilburi, V.

toast (¿brindis, o tostada?), ?, i. Tobralco (un tejido; abr. de Total Broadhurst Lee Co.), V.

tráfico (circulación), c, p, V.

trailer (avance de pelicula), i.

transformador (¿de transformer?), ?.

transistor (invención norteanin.), p. transmisor (¿de transinitter?), ?. traveling (término cinem.), i. tren (anglicismo), D.

trench coat (cn los escaparates).

trinchera (en los escaparates).

trole, trolebús, c, V.

Truman: gafas, camisas, corbatas - i. 
trust, por ej.: Trust Joyero (cmpresa) $i, V$.

túnel, tr. V.

turf: - match (deporte, carreras), $p$.

turista, turismo, V.

Tyron Power: un -, i.

última palabra (novisimo), ?, c. vagón (de wagon, carro de 4 ruedas), $\mathrm{V}$. vatio (de $W$ att).

very well (muy bien), c.

vitamina, ?.

wasinton (de Washington): na-

ranja, popularmente: guasin, $c$. wat, vatio, de Watt, inventor escocés.

wáter clóset (pop., wáter), V.

week-end, i.

welter (boxeo), i.

whisky, c, V.

whist, $\mathrm{V}$.

wrestling (lucha libre), i.

yachting club, i.

yanqui (Yankec, de Nueva Inglaterra o del norte de Estados Unidos).

yarda (de yard). yate (yacht), V. yola (yarol), V.

HOWARD STONE. 